\title{
OS DIREITOS FUNDAMENTAIS E A PANDEMIA COVID-19 - O CIDADÃO DEFICIENTE E O TERCEIRO SECTOR
}

\author{
FUNDAMENTAL RIGHTS AND THE PANDEMIC \\ COVID 19 - THE DISABLED CITIZEN AND THE THIRD SECTOR
}

Pedro Trovão do Rosário ${ }^{1}$

\begin{abstract}
Resumo: Face à situação epidemiológica global havendo todas as fragilidades, inclusivamente as expressas pela Organização Mundial de Saúde (OMS), sendo esta uma oportunidade de avaliação e melhoria, pondere-se a ação do Estado e a necessidade de recurso ao terceiro sector, ou Instituição Particulares de Solidariedade Social (IPSS), as quais asseguram o cumprimento e a efetivação de direitos fundamentais como o direito à saúde, o direito à educação, à proteção na infância e na terceira idade, sendo essenciais ao cidadão deficiente. Em momentos de crise o terceiro setor (IPSS) é uma economia que se comporta de forma expansionista e em contraciclo, assegurando a concretização de direitos fundamentais. A par, como com o cidadão deficiente, há que aprender com a sua experiência em situação de diversidade constante e potenciar a sua resiliência. Impõe-se uma definição legal clara, longe do livre arbítrio do Estado no papel das IPSS e, no quadro deste estudo, ao modo de proteção dos cidadãos portadores de deficiência, como da generalidade das IPSS em momento de uma crise pandémica onde uma percentagem relevante dos cidadãos se encontram em particular vulnerabilidade.As IPSS, tantas delas centenárias, tantas temáticas, locais ou regionais, numa relação de proximidade com os cidadãos e com as famílias, devem ser protagonistas neste processo de reconstrução da sociedade, onde o Estado assegurará a inclusão da deficiência como um requisito em todas as ações e sistemas COVID-19.
\end{abstract}

Palavras-Chave: Deficiência, Direitos Fundamentais, Instituição Particulares de Solidariedade Social, pandemia.

\begin{abstract}
In view of the global epidemiological situation, with all the weaknesses, including those expressed by the World Health Organization, this is an opportunity for evaluation and improvement, considering State action and the need for recourse to the third sector, or Private Social Solidarity Institution (IPSS), which ensure the fulfillment and enforcement of fundamental rights such as the right to health, the right to education, protection in childhood and old age, being essential to disabled citizens.In times of crisis the third sector is an economy that behaves in an expansionist and countercyclical manner, ensuring the realization of fundamental rights. At the same time, as with the disabled citizen, it is necessary to learn from their experience in a situation of constant diversity and enhance their resilience. A clear legal definition is required, far from the free will of the State in the role of IPSS and, in the context of this study, the way of protecting citizens with disabilities, as in the generality of IPSS at the time of a pandemic crisis where a relevant percentage citizens are particularly vulnerable. The IPSS, so many of them centuries old, so many thematic, local or regional, in a close relationship with citizens and families, must be protagonists in this process of reconstructing society, where the State will ensure the inclusion of disability as a requirement in all COVID-19 actions and systems.
\end{abstract}

Keywords: Disability, Fundamental Rights, Private Social Solidarity Institution, pandemic.

\footnotetext{
${ }^{1}$ Pedro Trovão do Rosário, Doutor em Direito, Investigador do Centro de Investigação Ratio Legis - UAL, Professor Universitário - Universidade Autónoma de Lisboa (Rua de Sta. Marta, no 47 - 1150-293 Lisboa - Portugal; prosario@autonoma.pt); ) e Advogado na Trovão do Rosário \& Associados, Sociedade de Advogados, SP, RJ.
} 


\section{Introdução}

Por Decreto do Presidente da República Portuguesa n. ${ }^{\circ}$ 14-A/2020 de 18 de março, foi declarado em Portugal o estado de emergência, com fundamento na verificação de uma situação de calamidade pública.

Tal com fundamento - de facto- na qualificação pela Organização Mundial de Saúde em 11 de março de 2020, de uma situação "de emergência de saúde pública ocasionada pela doença COVID-19 como uma pandemia internacional, constituindo uma calamidade pública”.

O fundamento jurídico encontrou-se necessariamente nas disposições constitucionais (artigo $19^{\circ}$ ) e legais (Lei 44/86, de 30 de setembro), limitando-se assim ao estritamente necessário para a adoção das medidas correspondentes à suspensão do exercício de direitos, liberdade e garantias enunciados naquele Decreto. Desta forma, ficou parcialmente suspenso o exercício de direitos como direito de deslocação e fixação em qualquer parte do território nacional; de propriedade e iniciativa económica privada; direitos dos trabalhadores de circulação internacional; de reunião e de manifestação; de liberdade de culto; de resistência.

Mais se refere no Decreto que "as limitações de direitos, liberdades e garantias" tiveram "o respaldo Constitucional que só o estado de emergência pode dar, reforçando a segurança e certeza jurídicas e a solidariedade institucional": nos termos dos artigos $19 .^{\circ}, 134 .^{\circ}$, alínea d), e 138..$^{\circ}$ da Constituição e da Lei $n^{\circ} 44 / 86$, de 30 de setembro, alterada pela Lei Orgânica $n^{\circ} 1 / 2011$, de 30 de Novembro e pela Lei Orgânica n ${ }^{\circ}$ 1/2012 de 11 de Maio.

Tal refletindo, num Estado de Direito (artigo $2^{\circ}$ da Lei Fundamental Portuguesa) o estatuído no artigo $19^{\circ}$ da Constituição da República Portuguesa (C.R.P.) que " $n$ ” 1 - Os órgãos de soberania não podem, conjunta ou separadamente, suspender o exercício dos direitos, liberdades e garantias, salvo em caso de estado de sítio ou de estado de emergência, declarados na forma prevista na Constituição.”. Assim, desde já e por cautela necessária, distinguir a Declaração do estado de emergência de outras formas de atuação como a Declaração de estado de calamidade, ao abrigo do disposto na Lei de Bases da Proteção Civil.

Em Portugal foram, entretanto, adotadas medidas sob "o estado de calamidade", ao abrigo do disposto na Lei de Bases da Proteção Civil, Lei n. ${ }^{\circ}$ 27/2006, de 3 de Julho. Impõe-se ponderar o que tal Lei ordinária (ao contrário da Lei com o Regime do estado de sitio e do estado de emergência que reveste obrigatoriamente a forma de Lei orgânica - artigos $166^{\circ} / 2,168^{\circ} / 5,136^{\circ}$ / 2 e3, 112\% 3 da CRP- assim com valor reforçado e com a obrigação de aprovação por maioria absoluta dos deputados em efetividade de funções) prevê e assim perceber da sua aplicabilidade a uma situação de pandemia: estabelece o artigo 9\%/3 que "A situação de calamidade pode ser declarada quando, face à ocorrência ou perigo de ocorrência de algum ou alguns dos acontecimentos referidos no artigo 3 . $^{\circ}$, e à sua previsível intensidade, é reconhecida a necessidade de adotar medidas de caráter excecional destinadas a prevenir, reagir ou repor a normalidade das 
condições de vida nas áreas atingidas pelos seus efeitos”, prevendo por sua vez o mencionado (e limitador) artigo $3^{\circ}$ as Definições de acidente grave e de catástrofe, sendo “( $\left.n^{\circ} 1\right)$ Acidente grave é um acontecimento inusitado com efeitos relativamente limitados no tempo e no espaço, suscetível de atingir as pessoas e outros seres vivos, os bens ou o ambiente." e "2 - Catástrofe é o acidente grave ou a série de acidentes graves suscetíveis de provocarem elevados prejuízos materiais e, eventualmente, vítimas, afetando intensamente as condições de vida e o tecido socioeconómico em áreas ou na totalidade do território nacional."

Acaso uma situação “de emergência de saúde pública ocasionada pela doença COVID-19 como uma pandemia internacional, constituindo uma calamidade pública" se confunde com um acidente grave com efeitos limitados no tempo e no espaço, ou uma catástrofe (enquanto acidente grave ou série de acidentes graves)? Afigura-se claramente que não.

A questão acima referida adquire maior relevância no momento atual, em que se assistia já antes da pandemia a uma crise de valores relativamente ao Estado de Direito democrático e aos Direitos Fundamentais. A previsão constitucional (artigo 19²) relativa à Declaração do estado de sítio ou do estado de emergência não é uma norma habilitante, é uma norma restritiva do poder político. Claramente.

No vértice do sistema jurídico português, a Constituição da República Portuguesa prevê no seu artigo $71^{\circ}$ a igualdade de direitos e deveres dos portadores de deficiência, obrigando-se a par o Estado a "realizar uma política nacional de prevenção e de tratamento, reabilitação e integração dos cidadãos portadores de deficiência e de apoio às suas famílias". Mas, no plano infraconstitucional verifica-se uma ação tímida e sem evidência de um imperioso "mainstreaming", onde o Estado opta por legislar de forma casuística e não integrada, como ocorreu com o Decreto-lei 319/91, de 23 de agosto onde estabeleceu um "o regime educativo especial aplicável aos alunos com necessidades educativas especiais", com a Lei 38/2004, de 18 de agosto "as bases gerais do regime jurídico da prevenção, habilitação, reabilitação e participação da pessoa com deficiência", a Lei 46/2006 de 28 de agosto de 2006 que "proíbe e pune a discriminação em razão da deficiência e da existência de risco agravado de saúde" e em 2019 a Lei n. $^{\text {o }}$ 4/2019 de 10 de Janeiro com o "sistema de quotas de emprego para pessoas com deficiência, com um grau de incapacidade igual ou superior a $60 \%{ }^{2}$

A Convenção sobre os Direitos das Pessoas com Deficiência adotada pela ONU em 2006, assim como anteriormente a Convenção 159/83 da OIT têm o propósito de promover, proteger e assegurar o pleno exercício dos direitos humanos das pessoas com deficiência, impondo aos Estados-membros medidas legislativas, administrativas ou de qualquer outra natureza para a

\footnotetext{
2 ROSÁRIO, Pedro Trovão do, "Deficiência: Valorização jurídica das qualidades especiais" In "Direito Constitucional Luso e Brasileiro no âmbito da pacificação social" - ISBN 978-85-362-9343-1, Ed. Juruá - Santa Catarina-Brasil, Janeiro de 2020
} 
implementação dos direitos das pessoas deficientes. A Convenção sobre os Direitos das Pessoas com Deficiência vincula o Estado português por força do disposto nos artigos $8^{\circ}$, número 2 e $16^{\circ}$, número 1 da CRP, sendo aqui patente o primado do Direito Internacional e encontrarem-se vinculados os órgãos do Estado.

Se houve sistemas jurídicos, como o brasileiro, em que a Convenção Internacional sobre os Direitos das Pessoas com Deficiência foi o primeiro tratado internacional de direitos humanos aprovado nos termos do art. $5^{\circ}, \S 3 .^{\circ}$, da Constituição Federal, passando a ter estatuto constitucional, já outros como Portugal a despeito do artigo $8^{\circ}$ da Constituição da República Portuguesa demora a atuar cabalmente ${ }^{3}$.

Portugal, assim como outros países, ratificaram um documento denominado Convenção sobre os Direitos das Pessoas com Deficiência (CDPD) que tem como objetivo garantir a todas as pessoas portadores de deficiência "o pleno e igual gozo de todos os direitos humanos e liberdades fundamentais" ${ }^{4}$, assim como o respeito e a dignidade.

De acordo com os últimos dados estatísticos oficiais do Banco Mundial e da Organização Mundial de Saúde ${ }^{5}$, mais de mil milhões de pessoas no mundo encontra-se em situação de deficiência. Ou seja, um em cada sete seres humanos.

Em plena crise Covid 19, tendo sido o estado de emergência declarado em Portugal nos termos acima referidos, com barreiras para todos, estas assumem particular impacto nos cidadãos deficientes. Vejam-se as medidas de prevenção (desde logo no acesso à própria informação; na possibilidade de execução de muitas das recomendações, como acesso aos meios de higiene; mobilidade, com dependência do contato físico com o meio ambiente; dificuldade em manter distância social devido à dependência de pessoas de apoio ou porque sitas em instituições psiquiátricas ou noutras instituições, privadas de liberdade e em condições de pobreza e superlotação); barreiras às medidas de minimização e controle de riscos (como a implementação de quarentenas, toque de recolher ou programas restritivos similares que podem implicar a interrupção nos serviços vitais para muitas pessoas com deficiência e limitar o exercício de direitos básicos, como alimentos, lavagem e saneamento e comunicações, pois para muitas pessoas com deficiência o pessoal de suporte e assistência é absolutamente vital) e barreiras aos cuidados de saúde e medidas de atenção, pois se forem infetadas com COVID-19, as pessoas com deficiência enfrentarão barreiras adicionais na busca e obtenção de atendimento médico perante

3 ROSÁRIO, Pedro Trovão do, "Deficiência: Valorização jurídica das qualidades especiais" In "Direito Constitucional Luso e Brasileiro no âmbito da pacificação social” - ISBN 978-85-362-9343-1, Ed. Juruá - Santa Catarina-Brasil, Janeiro de 2020.

${ }^{4}$ Portugal. Convenção sobre os direitos das pessoas com deficiência. 2011. (Disponível em https://www.inr.pt/content/1/1187/convencao-sobre-os-direitos-das-pessoas-com-dificiencia) .

${ }^{5}$ Organización Mundial de la Salud (OMS) y Banco Mundial (BM), 2011. Disponivel em 9 de Abril de 2020, em : https://www.whoe.int/disabilities/world_report/2011/accessible_es.pdf?ua=1. 
os limites de comunicação e mobilidade. Estamos assim perante pessoas mais propensas a estar em risco em casos mais graves motivados por problemas de saúde relativos à deficiência. Igualmente é recorrente potenciar-se a discriminação e negligência em momentos de crise, desvalorizando-se a deficiência e o seu portador no acesso aos cuidados de saúde, estes, por natureza, escassos (em recursos e pessoal). Ou seja, se os deficientes já são habitualmente discriminados no exercício de direitos fundamentais, hoje o seu o direito à saúde, à integridade física e à vida são ainda mais claramente afetados.

As pessoas com deficiência são desproporcionalmente afetadas pelo COVID19. De acordo com a ONU, dos mil milhões de pessoas com deficiência, $80 \%$ vivem em países em desenvolvimento. Aqui os cuidados de saúde, as condições sanitárias (falta de acessibilidade à água, saneamento e higiene, tantas vezes com dependência de contato físico para obter apoio em instalações inadequadas), os sistemas de proteção social e a realidade jurídica, na efetivação de direitos fundamentais é em regra mais frágil. Assim, as pessoas com deficiência correm maior risco de contratação e de sofrerem um impacto maior do COVID-19.

De modo a assegurar respostas adequadas à inclusão das pessoas com deficiência, a ONU apresentou um conjunto de ações e recomendações, respeitando assim os princípios desenvolvidos nos demais instrumentos (por exemplo empowerment) ${ }^{6}$ aprovados neste domínio. Procede-se à análise do impacto da COVID- 19 sobre pessoas com deficiência, concluindo com a apresentação de ações e recomendações "para tornar a resposta e a recuperação inclusivas de pessoas com deficiência".

Para tal será essencial colocar a temática da deficiência em todas as medidas públicas de adequação à pandemia (mainstreaming), ou seja, criar uma combinação de medidas principais e específicas para a deficiência de modo a garantir a inclusão de pessoas com deficiência.

Simultaneamente há que garantir acessibilidade a informações, instalações, serviços e programas na resposta e na recuperação da COVID-19.

Numa situação de isolamento a pessoa com deficiência tem que possuir as ferramentas para tomar as decisões necessárias, estar em segurança e ter assegurado o acesso à saúde e serviços públicos.

Mantem-se a preocupação pelo empowerment, ou seja, garantir-se uma participação ativa das pessoas com deficiência e/ou seus representantes e organizações, pois são quem melhor conhece e consegue apresentar soluções para a melhor integração.

Finalmente, com base no documento da ONU há que estabelecer mecanismos de fiscalização e de responsabilização para garantir a inclusão da deficiência na resposta à COVID-19, assim acompanhando a execução das políticas necessárias.

${ }^{6}$ http://oddh.iscsp.ulisboa.pe/index.php/pt/2013-04-24-18-50-23/outras-publicacoes/item/454_onu_policybrief 
As pessoas com deficiência estão em maior risco de discriminação no acesso aos cuidados de saúde, sendo patente nos procedimentos de salvamento de vidas durante o surto. Nalguns países, aos serviços de saúde foram impostas decisões de racionamento, incluindo protocolos de triagem nas camas de terapia intensiva ou no acesso a ventiladores. Tal por referência a critérios discriminatórios, como a idade ou a deficiência?

Por outro lado, os serviços de saúde ao terem sido logo exauridos, o acesso aos cuidados de saúde, reabilitação e assistência para pessoas com deficiência foram de imediato prejudicados, ignorando o seu maior grau de necessidade.

Recorda ainda o estudo que as pessoas com deficiência são particularmente desfavorecidas num cenário socioeconómico mais débil, como é o que resulta do COVID-19 e das medidas para controlar a pandemia, assim no emprego e proteção social, na educação, nos serviços de suporte, e, claramente diminuindo a proteção e aumentando a da violência (ex. doméstica) nas pessoas com deficiências.

Propõe assim a ONU a criação de bases sustentadas nos direitos humanos para a resposta à COVID-19 associadas à deficiência numa ótica de mainstreaming, elemento estruturante das propostas na área a deficiência que ora adquirem particular relevo, pois as necessidades dos cidadãos deficientes são comuns às dos demais cidadãos, mas com especificidades e aspetos de necessária adequação consoante cada realidade (proteção da saúde, serviços básicos, habitação, circulação, etc.). Ou seja, aquando da conceção das propostas nas diversas áreas para a generalidade dos cidadãos há sempre que ter presente a necessidade da sua adequação a todos os cidadãos e assim a inclusão e integração da deficiência em todos planos e esforços. Assim, há que (a) assegurar a não discriminação na resposta e na recuperação ao COVID-19 com base na deficiência, bem como quaisquer opções ou critérios nas políticas a definir que possam ter um impacto desproporcional nas pessoas com deficiência. Ponderada a (b) circunstância de as pessoas com deficiência enfrentarem uma discriminação intersecional e múltipla em consequência entre outros fatores de desigualdade como o género, idade, etnia, raça, orientação sexual, origem, sentindo um maior impacto da COVID- 19 na sua condição económica e social, há que estabelecer logo num primeiro momento um compromisso de que haverá um necessário cuidado na resposta para as formas múltiplas e cruzadas de discriminação enfrentadas por pessoas com deficiência. Como terceira preocupação (c) há que garantir em igualdade a acessibilidade a instalações, serviços e informação ao cidadão deficiente (informação em saúde pública, edifícios, transportes, comunicações, tecnologias, bens e serviços), pois só assim as pessoas com deficiência podem tomar quotidianamente as decisões necessárias, viverem independentes e até isoladas se em quarentena, bem como aceder a serviços públicos e de saúde. Igualmente, (d) as

\footnotetext{
${ }^{7}$ Baart \& F. Taaka (2017), "Barriers to Healthcare Services for People with Disabilities in Developing Countries: A Literature Review”, Disability, CBR \& Inclusive Development, 28(4). - disponível em: http://dcidj.org/article/view/656
} 
pessoas com deficiência têm o direito de participar plena e efetivamente nas decisões, no planeamento, na implementação e no acompanhamento da execução de políticas que afetam as suas vidas (empowerment), sendo múltiplas as situações de deficiência e as questões daí resultantes, pelo que o envolvimento ativo das pessoas com deficiência e/ou dos seus representantes, como organizações para garantir uma resposta inclusiva.

As Parcerias e colaboração melhorarão a eficácia e a responsabilidade, com inclusão direta, como adiante se desenvolverá. A (e) prestação de contas é essencial para garantir que a resposta e recuperação do COVID-19 são inclusivas e respeitam(aram) os direitos das pessoas com deficiência. Finalmente, (f) a análise e decomposição de dados vai ser uma oportunidade para se compreender as diferentes maneiras pelas quais as pessoas portadores de deficiência, em cada deficiência, reagem e vivenciam fenómenos como a COVID-19.

Igualmente integradas no "resumo de políticas" da ONU encontramos ações e recomendações sectoriais, como na Saúde, nas instituições onde vivem, nos serviços de apoio à vida diária, na proteção social e no emprego, na educação, na prevenção da violência e em situações de assistência humanitária.

Ora destacamos a importância da situação das pessoas com deficiência institucionalizadas e perante a educação, pelos motivos que adiante se exporão.

A institucionalização é uma realidade comum nas diversas sociedades onde para apoio ao cidadão deficiente e suas famílias se foram encontrando modelos públicos e privados para acolhimento e acompanhamento daqueles cidadãos.

Por seu lado, o direito à educação é um direito fundamental, assim como a liberdade de apender e de ensinar, mesmo que se possa querer coloca-las em gerações ou dimensões diversas dos direitos fundamentais. Este direito à educação é, tantas vezes, assegurado no que diz respeito ao cidadão deficiente por instituições privadas (associações, cooperativas, fundações), tantas vezes com estatuto de utilidade pública, expressão do "terceiro setor": Instituições Particulares de Solidariedade Social - IPSS.

Estatui a Constituição da República Portuguesa (CRP) a sua existência no seu artigo 63\%/5, existindo em Maio de $2020^{8}$ aproximadamente cinco mil e cem IPSS, a acrescer mais de cem Casas do Povo e quase duzentas cooperativas. Tal é significativo da implantação deste modelo de solidariedade, de economia social.

De referir que a ação das IPSS é acompanhada pela tutela, bem como pela Confederação Nacional das Instituições de Solidariedade (CNIS) ${ }^{9}$. Estas, são constituídas por iniciativa de particulares, sem fins lucrativos, com o propósito de dar expressão organizada ao dever de solidariedade e de justiça entre os indivíduos, não sendo administradas pelo Estado ou por

\footnotetext{
${ }^{8} \mathrm{http}$ /www.seg-social.pt/publicacoes?bundleld=16414310, disponível em 18.VII.2020

${ }_{9}$ https://cnist.pt/cnis-3, disponível em 18.VII.2020.
} 
outro ente público. Assim encontramos IPSS para fins como apoio a crianças e jovens, proteção dos cidadãos na velhice e invalidez, promoção e proteção da saúde, nomeadamente através da prestação de cuidados de medicina preventiva, curativa e de reabilitação, educação e formação profissional dos cidadãos, entre outras valências sociais. A sua ação é acompanhada pelo Estado, inclusivamente havendo a obrigação pelas IPSS (e suas equiparadas) de um dever de envio à tutela das suas contas anuais (vide Decreto-Lei n. ${ }^{\circ}$ 172-A/ 2014 de 14 de Novembro, que aprova o Estatuto das IPSS).

Ora, uma larga percentagem de cidadãos dependem do funcionamento das IPSS, diretamente e indiretamente: as famílias, como os próprios utentes. No caso dos cidadãos deficientes tal importância é por demais óbvia. Mas, o Estado (legislador) afirmou no preambulo do seu Estatuto das Instituições Particulares de Solidariedade Social (o Decreto-Lei n. ${ }^{\circ}$ 172A/2014 procedeu à quinta alteração ao Decreto-Lei $n^{\circ} 119 / 83$, de 25 de fevereiro) do qual se transcreve: "Volvidos 31 anos após a publicação do Decreto-Lei n. ${ }^{\circ} 119 / 83$, de 25 de fevereiro, o setor social e solidário, representado pelas misericórdias, instituições de solidariedade social e mutualidades, vulgo Instituições Particulares de Solidariedade Social (IPSS) tem assumido uma posição de enorme preponderância no estabelecimento e desenvolvimento de um conjunto de respostas sociais, em todo o território nacional, alicerçado no quadro axiológico da solidariedade social e desenvolvendo-se num modelo de atuação que é revelador de uma abordagem mais humanista, mais próxima, menos dispendiosa para o Estado e mais benéfica para os cidadãos. Este setor, ao longo destas três décadas, não só cresceu exponencialmente em número de IPSS constituídas, como fundamentalmente passou a assumir, na nossa sociedade, uma importância social e económica de elevado relevo junto das comunidades em que as instituições estão inseridas, por via da sua atuação. Com efeito, a ação de solidariedade social exercida pelas IPSS não se encerra, apenas, no setor da segurança social, abrangendo igualmente outros domínios, como a saúde, a educação ou, ainda, em áreas em que as necessidades sociais dos indivíduos e das famílias encontram apoio e resposta. Por via da sua proximidade junto da sociedade, as IPSS têm demonstrado, pela sua ação, possuir capacidade para responder com elevada eficácia às situações de emergência social e de apoio aos cidadãos em situação de maior vulnerabilidade. A promoção solidária destas mesmas instituições constitui, assim, a raiz da sua ação, assente na capacidade de inovação e adaptação, face às respostas sociais necessárias.”.

Porque a ação das IPSS não se sente apenas nos utentes e nas suas famílias, na mesma norma, o Estado reconhece que "importa referir que, para além da importância que o setor social e solidário possui no apoio aos cidadãos, as IPSS adquirem uma outra e especial importância na dinamização das economias locais onde estão implementadas, constituindo-se, assim, como agentes da dita economia social. A sua capacidade de dinamização económica e social é, hoje, reconhecida e valorizada por todos, desde logo, pelo peso que possui no emprego em Portugal, cerca de $5,5 \%$, e porque em momentos de crise é uma economia que se comporta de forma 
expansionista e em contraciclo, quando comparado com os outros setores tradicionais da economia. Uma economia que é das pessoas, para as pessoas e que respeita as comunidades.

É de justiça salientar que, por via de uma iniciativa inovadora, em termos internacionais, o Governo em parceria com o Instituto Nacional de Estatística, I. P., desenvolveu a Conta Satélite da Economia Social, a qual, pela primeira vez e de forma rigorosa, retratou todo este setor, dando-lhe uma visibilidade e importância económica que até então não possuía. Hoje, sabemos, em concreto, que este setor possui uma dimensão tão ou mais importante do que outros setores tradicionais da nossa economia, não apenas pelo universo de 55 mil organizações que o constituem, pelas 227 mil pessoas que emprega, como também por ser responsável por 5,5\% do emprego remunerado nacional e por 2,8 \% do Valor Acrescentado Bruto".

Tal a realidade das IPSS: essencial para os utentes e suas famílias, na concretização de direitos fundamentais, essencial ao Estado (em sentido lato) pois atua onde seria competência desde, é um motor económico criador de empregos e de riqueza e atua em contraciclo: "em momentos de crise é uma economia que se comporta de forma expansionista e em contraciclo".

Face à situação epidemiológica global havendo todas as fragilidades, inclusivamente as expressas pela OMS, sendo esta uma oportunidade de avaliação e melhoria, pondere-se a ação do Estado. Tal, com o cuidado de as IPSS assegurarem o cumprimento, a efetivação de direitos fundamentais como o direito à saúde (artigo 64 ${ }^{\circ}$ da CRP e assim do direito à vida e à integridade física e moral - artigos $24^{\circ}$ e $25^{\circ}$ da CRP), o direito à educação $\left(73^{\circ} 74^{\circ}, 75^{\circ}\right.$, ) proteção na infância e na terceira idade $(67 / 2 / \mathrm{b})), 69^{\circ}$ e $72^{\circ}$, todos da CRP), entre tantos outros.

Ora, nesta avaliação, há que fazer uma profunda reflexão do momento atual . Que "em momentos de crise é uma economia que se comporta de forma expansionista e em contraciclo", assegurando a concretização de direitos fundamentais.

Tem-se presente que foi publicada em Portugal a Portaria n. ${ }^{\circ} 85-\mathrm{A} / 2020$, de 03 de abril, com objetivo de apoiar e agilizar a ação das Instituições Particulares de Solidariedade Social e entidades equiparadas e assim, no seguimento deste diploma, e para efeitos do previsto no n. $^{\circ} 2$ do artigo $18 .^{\circ}$ do Decreto-Lei n. ${ }^{\circ} 24$-A/2020, de 29 de maio, foi prorrogado, até 31 de outubro, o prazo para apresentação das contas relativas ao ano de 2019.

Impõe-se uma definição legal clara, que não fique num plano limite de livre arbítrio do Estado relativamente às IPSS e, no quadro deste estudo, ao modo de proteção dos cidadãos portadores de deficiência, como da generalidade das IPSS em momento de uma crise pandémica onde uma percentagem relevante dos cidadãos se encontram em particular vulnerabilidade.

As instituições que têm internados ou dão apoio a cidadãos deficientes tiveram que se recriar. Porque estão em causa cidadãos tantas vezes mais frágeis fisicamente (tantas vezes mais suscetíveis a condições secundárias como problemas pulmonares, diabetes, doenças cardíacas e obesidade, o que pode piorar o resultado de Infecções por COVID-19) e emocionalmente (em 
confinamento não podem contactar proximamente com familiares, ou sequer com os cuidadores), geram situações de maior tensão, conflitualidade e violência dentro das instituições. O próprio pessoal médico, de enfermagem, psicólogos, terapeutas, auxiliares, entre outros, tiveram que encontrar novas dinâmicas e que responder a novos desafios que agravaram o quadro existente. Tudo com a necessidade de as instituições reduzirem os riscos de infecção, abordando situações de superlotação, isolamento e distanciamento físico medidas entre residentes, restringindo e modificando as visitas, tendo que adquirir e manter equipamentos de proteção obrigatória, com adequação a condições de higiene. Não esquecendo que as IPSS garantem a muitas pessoas com deficiência serviços de apoio à vida diária nos seus locais de residência ou ao recebê-las nas suas instalações, com pessoal (médico, de enfermagem, psicólogos, terapeutas, auxiliares, entre outros...) que assegura assistência geralmente especializada (linguagem de sinais, interpretação táctil, serviços domésticos, entre outros) os quais devem atuar com medidas para reduzir a exposição potencial a COVID-19 durante a prestação de serviços. Outro campo importante de ação das IPSS é a educação, um direito fundamental que no caso da deficiência pode ser encarado em simultâneo como garantia de integridade moral, desenvolvimento da personalidade e integração social. sumariamente: dignidade da pessoa humana.

Os alunos com deficiência já enfrentavam barreiras que os demais não encontravam. Sendo, conforme acima reconhecido pela ONU prejudicado o exercício de direitos fundamentais por este conjunto mais frágil de cidadãos, os quais ora sentirão dificuldades acrescidas relativamente aos demais no acesso ao ensino.

O ensino deve ser garantido, seja este presencial ou à distância e acessível a todos, por forma a garantir a continuidade da aprendizagem dos alunos com deficiência, como dos demais e, assim o retorno aos programas escolares. Tal, com o conhecimento específico, de proximidade, com a experiência das IPSS, potenciando o fornecimento de serviços especializados, equipamentos para apoiar a aprendizagem, com tecnologia e dispositivos de assistência e apoio aos cuidadores ou pais das crianças e jovens com deficiência.

$\mathrm{O}$ encerramento das escolas motivará o recurso a IPSS, que devem ser apoiadas para acesso a prestação de serviços complementares, como exames alimentares e médicos ou encaminhamento de mecanismos de abuso e negligência. Esta proximidade, o conhecimento próximo e livre que as IPSS têm do cidadão deficiente deve ser assim valorizado. Muitas pessoas com deficiência têm experiência de prosperar em situações de isolamento e com acordos de trabalho alternativos que podem proporcionar modelos para a necessária adequação à situação atual. As perspetivas e experiências do cidadão deficiente, a sua resiliência e capacidade de superação, o recurso a meios que permitem ultrapassar a adversidade, toda essa criatividade, permitirão novas abordagens e soluções inovadoras para os desafios. Assim, a COVID-19 pode ser uma oportunidade, de valorização do deficiente e das instituições que com este e para este trabalham: as IPSS. 
Com a crise, presente e futura, resultante do COVID-19 as medidas a tomar devem respeitar o mainstreaming e o empowerment, devem ser uma oportunidade na construção de mais igualdade, inclusão e sustentabilidade em economias e sociedades mais justas e resilientes perante as crises e pandemias que surgirão no futuro.

A própria ONU no texto citado valoriza o apoio às iniciativas locais e organizações da sociedade civil com a necessidade de promover, financiar e monitorar a inclusão de pessoas com deficiência em todas as etapas do projeto e implementação das medidas relevantes. Para tal o papel das IPSS, tantas delas centenárias como as Misericórdias, tantas temáticas em função da deficiência e outras ainda locais ou regionais, numa relação de proximidade com os cidadãos e com as famílias as quais integram tantas vezes os órgãos sociais das instituições, devem ser protagonistas neste processo de construção de uma nova fase. A par, o Estado assegurará que a inclusão da deficiência deve ser um requisito em todas as ações e sistemas COVID-19.

\section{Referências}

ANDRADE, José Carlos Vieira de Andrade - Os Direitos Fundamentais na Constituição de 1976. Coimbra: Almedina. ISBN 978-972-40-4669-3. p. 62, 2016.

ARAÚJO, António de - “Cidadãos Portadores de Deficiência - O seu lugar na Constituição da República”, ed. Coimbra Editora, Coimbra. ISBN 9789723210279, 2001.

BAART \& F. Taaka (2017), "Barriers to Healthcare Services for People with Disabilities in Developing Countries: A Literature Review", Disability, CBR \& Inclusive Development, 28(4).-disponível em: https://dcidj.org/article/view/656.

CANOTILHO, J.J. Gomes - Direito Constitucional e Teoria da Constituição. 7. a . ed. 6 reimp. Coimbra: Almedina. ISBN 978-972-40-2106-5, 2003.

CLAUDINO, Adelaide do Amparo Duarte - "A Orientação para a Formação Profissional de Jovens com Deficiência Intelectual". Editor: Secretariado Nacional para a Reabilitação e Integração das Pessoas com Deficência. Lisboa, (Livros SNR nº11). ISBN 972-9301-23-9, 1997.

MIRANDA, Jorge. Manual de Direito Constitucional: Direitos Fundamentais, tomo IV, 4. ${ }^{a}$ edição, Coimbra Editora, 2008.

NOVAIS, Jorge Reis - A Dignidade da Pessoa Humana. V. II. Coimbra: Almedina. 2017. ISBN 972-32-0851-2. p. 169. 
Organización Mundial de la Salud (OMS) y Banco Mundial (BM), 2011; https;//www.whoe.int/ disabilities/word report/2011/accessible es.pdf?ua=1. https;/www.oddh.iscsp.ulisboa.pt/ index.php/pt/2013-04-24-1850-23/outras-publicacoes/item/454-onu policybrief, disponível em 18.VII.2020.

ORNELAS, José; MONTEIRO, Fátima; MONIZ, M. João; DUARTE, Teresa - "Participação e Empowerment das Pessoas com Doença Mental e seus Familiares”, AEIPS Edições- Lisboa, 2005.

PINTO, Paulo Mota. O Direito ao Livre Desenvolvimento da Personalidade, in Boletim da Faculdade de Direito de Coimbra, Portugal-Brasil ano 2000, Coimbra Editora, 1999.

Portugal. Convenção sobre os direitos das pessoas com deficiência. 2011. (Disponível em: https://www.inr.pt/content/1/1187/convencao-sobre-os-direitos-das-pessoas-com-deficiencia).

ROSÁRIO, Pedro Trovão do. "Deficiência: Valorização jurídica das qualidades especiais” In "Direito Constitucional Luso e Brasileiro no âmbito da pacificação social" - ISBN 978-85-3629343-1, Ed. Juruá - Santa Catarina-Brasil, Janeiro de 2020.

ROSÁRIO, Pedro Trovão do. “Cidadania e Deficiência” in Jurismat VI, Portimão, 2015.

SAMPAIO, José Adércio Leite. Direitos Fundamentais. 2. ${ }^{\text {a }}$ Ed. Belo Horizonte: Del Rey. ISBN 978-85-384.0109-4.p. 228. 2010. 